(C) 2016, Elsevier. Licensed under the Creative Commons Attribution-NonCommercial-NoDerivatives 4.0 International

http://creativecommons.org/licenses/by-nc-nd/4.0/

\title{
Impact of amplitude jitter and signal-to-noise ratio on the nonlinear spectral compression in optical fibres
}

\author{
Sonia Boscolo $^{1}$, Julien Fatome ${ }^{2}$, and Christophe Finot ${ }^{2, *}$ \\ ${ }^{1}$ Aston Institute of Photonic Technologies, School of Engineering and Applied Science, Aston \\ University, Birmingham B4 7ET, United Kingdom \\ ${ }^{2}$ Laboratoire Interdisciplinaire Carnot de Bourgogne, UMR 6303 CNRS-Université de \\ Bourgogne-Franche-Comté, 9 avenue Alain Savary, BP 47870, 21078 Dijon Cedex, France \\ * Corresponding author: \\ E-mail address: christophe.finot@u-bourgogne.fr \\ Tel.: +33380395926
}

\begin{abstract}
We numerically study the effects of amplitude fluctuations and signal-to-noise ratio degradation of the seed pulses on the spectral compression process arising from nonlinear propagation in an optical fibre. The unveiled quite good stability of the process against these pulse degradation factors is assessed in the context of optical regeneration of intensity-modulated signals, by combining nonlinear spectral compression with centered bandpass optical filtering. The results show that the proposed nonlinear processing scheme indeed achieves mitigation of the signal's amplitude noise. However, in the presence of a jitter of the temporal duration of the pulses, the performance of the device deteriorates.
\end{abstract}

(C) 2016 Elsevier

Keywords: Nonlinear spectral compression, optical regeneration, nonlinear fiber optics 


\section{Introduction}

Self-phase modulation (SPM) in optical fibre is ordinarily associated with spectral broadening of an ultra-short optical pulse. However, for appropriate initial conditions of the input pulse, SPM can result in significant spectral compression [1]. Indeed, SPM causes spectral compression or broadening depending on the initial frequency modulation (chirp) of the pulse electric field. Specifically, a pulse with a negative chirp, such as that imparted by an anomalously dispersive element, is compressed by the effects of SPM [2-4]. This method of spectral compression has been implemented using various types of fibres [5-7] and has also been theoretically investigated in centimeter-long nonlinear waveguides [8]. It is suitable for a very large range of wavelengths including Ti:sapphire wavelengths [5], the widely used 1- $\mu \mathrm{m}[7,9,10]$ and 1.55- $\mu \mathrm{m}$ [11] windows and the emerging $2-\mu \mathrm{m}$ band [12]. The process can also sustain simultaneous amplification of the pulse $[9,10,12]$, thereby providing an attractive solution to convert ultrashort pulses delivered by femtosecond oscillators into high-power, near-transform-limited picosecond pulses, and to counteract the spectrum expansion that usually occurs with the direct amplification of picosecond structures. The concept can be extended to cascade amplifier systems in which pulse spectrum narrowing allows its efficient amplification in the subsequent stages [13], and fibre laser cavities where spectral compression components have been employed in the model of an efficient similariton laser [14].

Several recent theoretical and experimental studies [7, 11, 15-17] have been concerned with the reduction of the intensity level of the residual side lobes in the compressed spectrum that, in the nonlinearity-dominant regime of propagation, result from the mismatch between the initial linear chirp of the pulse and the SPM-induced nonlinear chirp. However, to date, the problem of the stability of the spectral compression process against various forms of quality degradation of the initial pulses, such as amplitude fluctuations or broadband noise, has not been yet considered to our knowledge. In this paper, after recalling the principle of SPM-driven spectral compression and describing the operational parameters of the system, we analyze the impact of amplitude jitter and degraded optical signal-to-noise ratio (OSNR) of the seed pulses on the compression process by means of extensive numerical simulations. Remarkably, our results show that the spectral compression is rather stable against these pulse degradation factors, thereby revealing its potential for use in the context of all-optical regeneration. The computed 
intensity transfer function (TF) associated with the process is indeed shown to achieve both reduction of the amplitude fluctuations and improvement of the extinction ratio of an intensity modulated signal. However, the amplitude noise rejection ability of the device diminishes for operation at degraded OSNR.

\section{Model and situation being investigated}

Our numerical simulations of the spectral compression process in a fibre system are based on the standard nonlinear Schrödinger equation (NLSE) [18]:

$$
i \frac{\partial \psi}{\partial z}+\gamma|\psi|^{2} \psi-\frac{1}{2} \beta_{2} \frac{\partial^{2} \psi}{\partial t^{2}}=0
$$

where $\psi(z, t)$ is the complex electric field envelope, $z$ is the propagation distance, $t$ is the reduced time, $\beta_{2}$ is the group-velocity dispersion (GVD) parameter and $\gamma$ is the coefficient of cubic nonlinearity of the fibre. This equation neglects the effect of fibre loss, as well as higher-order linear and nonlinear effects. Although these effects can have noticeable impact on pulses shorter than 1ps, here we neglect them as the leading-order behavior is well approximated by Eq. (1).

Contrary to some recent studies of spectral compression in fibre that have used incoherent initial conditions [19, 20], we consider a transform-limited pulse with a Gaussian intensity profile $\psi_{0}(t)=\sqrt{P_{0}} \exp \left(-t^{2} / 2 T_{0}^{2}\right)$ as the initial condition for our study. The parameters $T_{0}$ and $P_{0}$ are a characteristic temporal value (the half-width at 1/e-intensity point in the case of a Gaussianshaped pulse) and the peak power of the initial pulse, respectively, and can be used to normalize the problem being studied [16]. The linear negative temporal chirp required for spectral compression to take place can be imprinted onto the initial pulse by stretching the pulse in the temporal domain in an anomalously dispersive medium, such as a pair of diffraction gratings [21, 22], a prism pair [3, 5], a fibre Bragg grating, or a segment of hollow core [9] or standard [11] fibre with anomalous GVD (and very low nonlinearity). As a result of GVD (described by Eq. (1) with $\gamma=0$ ), the different spectral components of the pulse acquire a frequency dependent delay. Even though such phase changes do not affect the pulse spectrum, they lead to temporal 
broadening of the pulse and a time dependence of the pulse phase. If $A$ denotes the temporal broadening factor, the peak power of the pulse will drop by the same factor $\left(P_{1}=P_{0} / A\right)$ owing to the conservation of energy. Moreover, in the far-field regime (i.e., when $A \gg>1$ ) the temporal phase becomes parabolic. The so obtained negatively linearly chirped pulse is then spectrally compressed in a nonlinear fibre.

For the purpose of illustration, we consider here an initial pulse with a full-width at half maximum (FWHM) duration of 2 ps at the wavelength $1550 \mathrm{~nm}$. After dispersive propagation, the pulse is stretched by a factor of $A=20$ and has a peak power of $P_{1}=2 \mathrm{~W}$. Nonlinear spectral compression of the pulse is then achieved through propagation in a highly nonlinear fibre (HNLF) with the Kerr coefficient $\gamma=10 \mathrm{~W}^{-1} \mathrm{~km}^{-1}$ and a low GVD coefficient of $\beta_{2}=1 \mathrm{ps}^{2} \mathrm{~km}^{-1}$. With such parameters that are typical of various demonstrations of spectral compression due to SPM in fibre [11], the nonlinearity-dominant regime of propagation is applicable [16].

Figure 1 illustrates the pulse evolution over a fibre length of $1250 \mathrm{~m}$. Significant spectral compression occurs in the fibre, followed by splitting of the pulse spectrum into an increasing number of substructures (Fig. 1(a)). The narrowing of the pulse spectrum is quantified with the spectral compression factor $C_{\text {fwhm }}$ (Fig. 1(b1)) defined as the ratio between the spectral FWHM width at some distance in the fibre and the entrance of the fibre. We also assessed the quality of the pulse spectrum by computing the spectral brilliance of the pulse at its central frequency (Fig. 1(b2)). The highest brilliance in the central region of the pulse spectrum is achieved at the propagation distance $z_{C}=750 \mathrm{~m}$, featuring a close to eightfold increase with respect to its value at the fibre entrance. The corresponding compressed spectrum is plotted in Fig. 2(a). The spectral compression factor is above 10 at $z_{\mathrm{C}}$. The optimum propagation distance $z_{\mathrm{C}}$ in terms of spectrum quality differs from both the distance $z_{\mathrm{F}}$ at which the temporal phase of the pulse becomes flat near the pulse center [16], and the distance of maximum spectral compression factor where the compressed spectrum is impaired by significant pedestals containing a non-negligible part of the pulse energy. The abrupt drop of $C_{\text {fwhm }}$ after the distance at which it is at its maximum is due to an increase in the intensity level of spectral satellites above the $-3 \mathrm{~dB}$ threshold used for its computation. The pulse spectrum splits up shortly after the distance of maximum $C_{\mathrm{fwhm}}$ and, at a distance of around $1100 \mathrm{~m}$ energy is no longer present in the vicinity of the central frequency. 

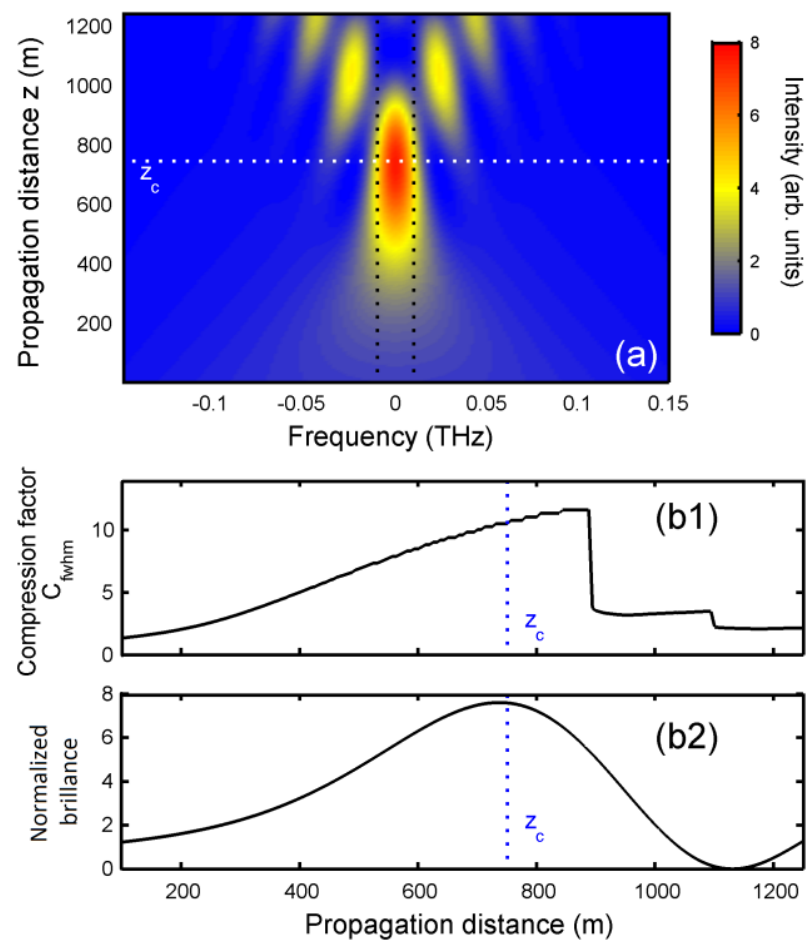

Figure 1: Spectral compression of an initial picosecond Gaussian pulse with the stretching factor $A=20$ in a HNLF. Longitudinal evolution of: (a) the spectral intensity profile, (b) the FWHM spectral compression factor and the spectral brilliance at the central frequency. The spectral brilliance is normalized to its initial value.

\section{Impact of initial amplitude jitter and OSNR}

In this section, we describe how the spectral compression process is affected by factors that may deteriorate an initial train of pulses. Hereafter, we will consider the optical spectrum obtained at the propagation distance of optimum compression $z_{\text {c }}$. First, we studied the effect of fluctuations in pulse peak power. To this end, we considered 1000 individual input pulses with the peak power $P_{1}$, and we superimposed a random independent peak power fluctuation to each of them governed by the $n$-th extraction of a Gaussian random variable with a relative standard deviation (or root-mean-square (rms) variation) of 8\%. In Fig. 2(a), we compare the average spectrum with the spectrum obtained in the absence of input fluctuations. We can see that the results are very close to each other, indicating that amplitude jitter does not heavily impact the average spectral behavior. However, when the spectra of individual pulses are overlapped together (Figs. 2(b1) and 2(c)), it appears that the different regions of the spectrum are not equally affected by the 
initial jitter of the pulses: while the central part of the spectrum turns up to be very stable, the spectral wings suffer from high fluctuations. The relative standard deviation of the output spectral intensity varies from $8 \%$ in the central lobe up to $120 \%$ in the side lobes. Such frequency dependence of a nonlinearly reshaped spectrum in normally dispersive fibres has been described in the context of super-continuum generation for wavelength-division multiplexed pulse sources [23], but it has never been discussed in connection with the process of spectral narrowing. We ascribe the high sensitivity of the spectrum edges to input pulse power fluctuations to the fact that non-negligible pedestals develop in the spectrum at $z_{\mathrm{C}}$ because of spectral interference between different temporal pulse parts having the same frequency [16]. This is confirmed by the results obtained at the distance $z_{\mathrm{F}}$ of chirp cancellation near the pulse center (Fig. 2(b2)). The monotonic temporal variation of the frequency chirp at $z_{\mathrm{F}}[16]$ restrains spectral interferences (Fig. 2(a), green curve), thereby leading to enhanced stability of the spectrum edges. However, fluctuations are more than twofold higher in the central lobe of the spectrum where most of the pulse energy is concentrated. This point will be discussed in the next section.

Next, we evaluated the effect of an OSNR degradation of the initial pulses. To this end, we considered pulses degraded by amplified spontaneous emission (ASE) noise whose power spectral density was assumed to be uniformly distributed within the spectral window in use, for the sake of simplicity and generality of the discussion. Our numerical model used a 2-ns temporal window combined with a spectral window of $4 \mathrm{THz}$. The energy of the pulses was maintained at a fixed value, corresponding to the peak power $P_{1}$, and the noise power was set accordingly to the desired OSNR. The averaged output spectra of 1000 pulses obtained for different input OSNR values $(10,20$ and $30 \mathrm{~dB})$ are displayed in Fig. 3(a), and show that the initial OSNR does not distort the average spectrum. This relaxes the need for an adjustment of the optimal compression distance. The output OSNR is up to $9 \mathrm{~dB}$ higher than the input one, which is fully consistent with the increase in spectral brilliance occurring at $z_{c}$ (Fig. 1(b2)). It is notable that no amplification of the noise level is observed in the spectrum. This accords with the absence of modulation instability phenomena in the normal-dispersion regime of scalar propagation in fibre [18]. The superposition of the spectra of the individual pulses confirms this trend (Fig. 3(b)). Fig. 3(c) shows the output OSNR as a function of the input OSNR, highlighting a fall-off in OSNR improvement for highly degraded input pulses. We partly attribute this fact to the increasing jitter of the temporal duration of the initial pulses that is observed with decreasing 
OSNR: as shown in Fig. 3(c3), a lower input OSNR brings about a higher relative standard deviation $\sigma_{\mathrm{D}} / M_{\mathrm{D}}$ of the probability distribution of the initial FWHM pulse duration. Consequently, as the SPM effect driving spectral compression depends on the temporal intensity gradient of the input pulses, it is inherently affected by fluctuations in initial pulse duration.
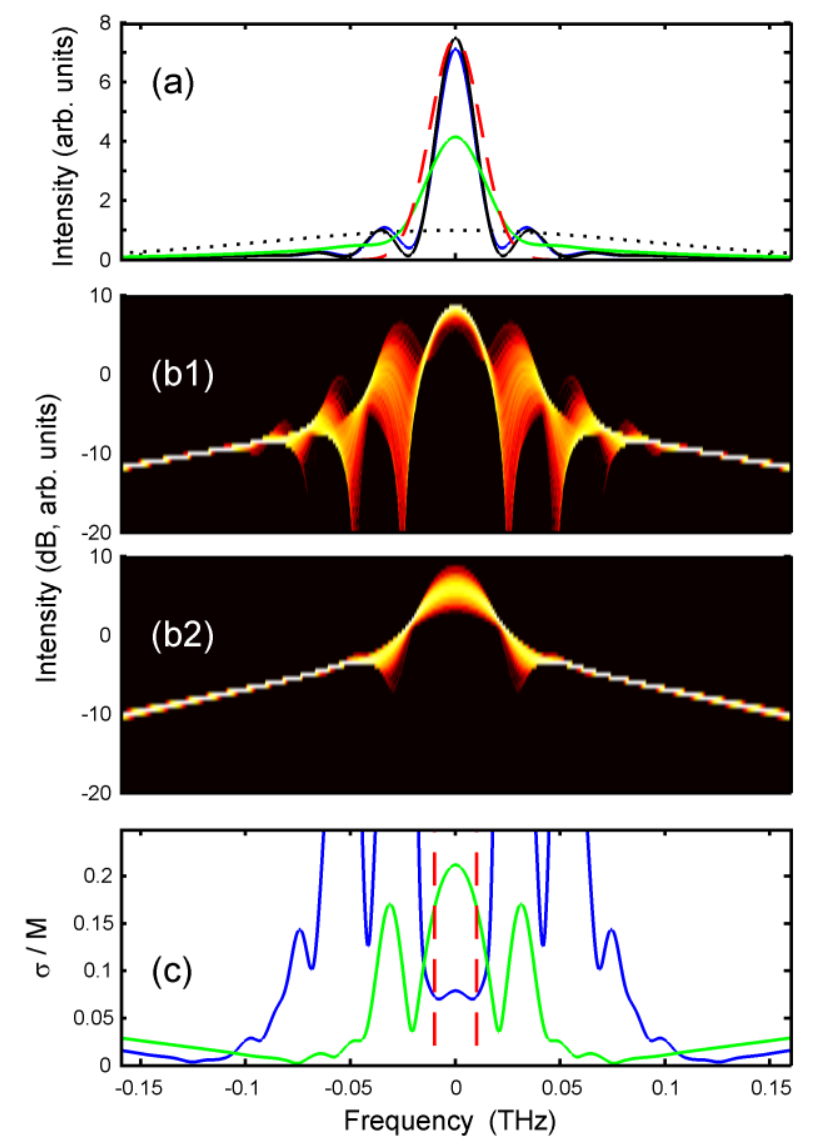

Figure 2: Impact of peak-power fluctuations of the initial pulses on the spectral compression process. (a) The averaged output spectrum of 1000 pulses at the optimum compression distance $z_{\mathrm{C}}$ (blue) is compared with the spectrum obtained in the absence of input fluctuations (black). Also shown are: the initial pulse spectrum in the absence of fluctuations (dotted black), the average spectrum at the distance of chirp cancellation near the pulse center $z_{\mathrm{F}}$ (green), and the transfer function of the spectral filter used in Section 4 (dashed red). (b) Overlap of the spectra of the individual pulses at $z_{\mathrm{C}}$ (subplot 1 ) and $z_{\mathrm{F}}$ (subplot 2). (c) Variation of the output spectral intensity $\sigma / M$ at $z_{\mathrm{C}}$ (blue) and $z_{\mathrm{F}}$ (green). The red dashed lines indicate the FWHM bandwidth of the spectral filter. 

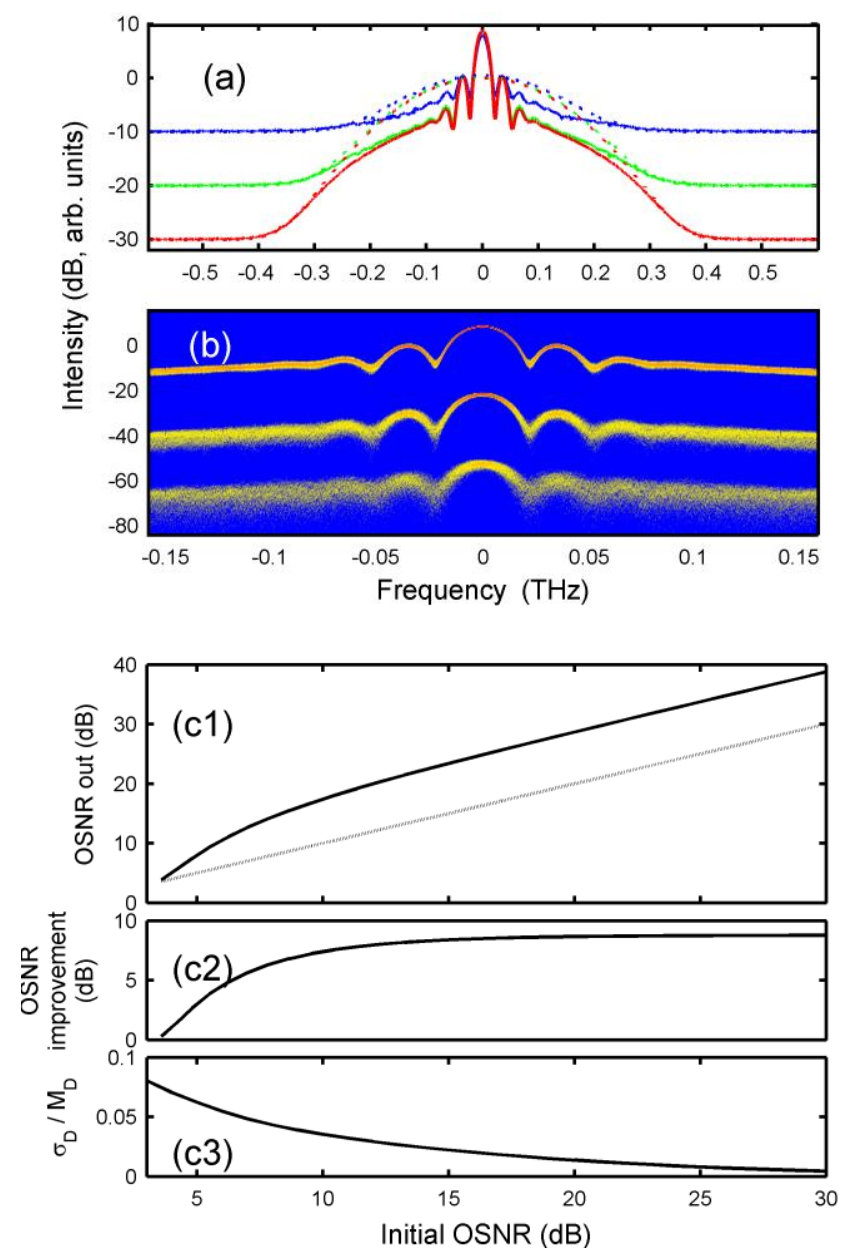

Figure 3: Impact of OSNR of the initial pulses on the spectral compression process. (a) The averaged output spectrum of 1000 pulses at $z_{\mathrm{C}}$ (solid) is compared with the input spectrum (dotted) for different input OSNR levels: $10 \mathrm{~dB}$ (blue), $20 \mathrm{~dB}$ (green) and $30 \mathrm{~dB}$ (red). (b) Overlap of the output spectra of the individual pulses for input OSNR levels of 10, 20 and $30 \mathrm{~dB}$ (plots from bottom to top). For clarity, the different plots have been offset by 30 $\mathrm{dB}$ along the vertical axis. (c) Output OSNR (subplot 1), OSNR improvement (subplot 2) and pulse width variation of the input pulses (subplot 3) versus input OSNR. The grey line in subplot 1 is a visual guideline representing the linear case of an output OSNR equaling the input one.

\section{Potentiality of optical regeneration}

The observed improvement in OSNR of the pulses after experiencing nonlinear spectral compression suggested us to evaluate the capability of the spectral compression process of being used in the context of optical regeneration of intensity-modulated signals. Several optical schemes utilizing nonlinear reshaping of optical spectra and optical bandpass filtering have been proposed and experimentally demonstrated. The method pioneered by P.V. Mamyshev [24, 25] 
relies on SPM in a normally dispersive fibre and subsequent frequency-offset spectral filtering to achieve optical pulse reshaping and re-amplification. The pulse dynamics occurring in an anomalously dispersive fibre can also be used in a combination with an optical bandpass filter (OBPF) centered at the carrier frequency of the signal to accomplish a power limiting function $[26,27]$. In our spectral compression scheme, we propose to place an OBPF centered at the carrier frequency after the HNLF to isolate the central part of the compressed spectrum, thereby converting the resulting change in central spectral brilliance into a change of the peak power of the temporal intensity profile. We used a Gaussian-shaped filter with a width at FWHM of 20 $\mathrm{GHz}$ in our study. We should note, however, that in opposition to the methods mentioned above, our scheme produces output pulses that are significantly longer than the pulses at the input to the system (before dispersive stretching). This restrains the cascading of several such devices, thereby limiting the device's use to the detection stage in a communication system where the temporally broadened waveform complies with the optoelectronic bandwidth of the photodetector.

A key parameter of the proposed device is the shape of its TF that nonlinearly maps the pulse peak power at the entrance of the HNLF $P_{\text {in }}$ to the peak power obtained after filtering $P_{\text {out }}$ (Fig. 4(a)). We can see that the TF features a rise and fall evolution and has a local maximum at $P_{\text {in }}=2 \mathrm{~W}$. This evidences that operation of the device near the peak of its TF should be beneficial to both the probability distributions of logical ones and zeros of a return-to-zero (RZ) signal in that the mark-to-space extinction ratio should be improved and the amplitude fluctuations in the one-bit pulses should be squeezed. It is noteworthy however a TF exhibiting a large plateau with an inflexion point would be preferable from a power equalization standpoint $[28,29]$. The shape of the TF also explains the amplification of the fluctuations in the central lobe of the pulse spectrum that are observed after propagation over the distance $z \mathrm{~F}$ in the fibre (Fig. 2(b2)). Given the scaling laws characterizing a close-to-purely nonlinear propagation (the power-distance product is the governing effect), the propagation of an initial pulse with a peak power of $2 \mathrm{~W}$ up to $z_{\mathrm{F}}$ is indeed equivalent to working with an initial peak power of $1.2 \mathrm{~W}$ and a fibre length $z_{\mathrm{C}}$. The $x$-intercept $P_{\mathrm{C}}$ of the tangent to the TF curve at the working power $P_{\text {in }}=1.2$ W satisfies the condition $0<P_{\mathrm{C}}<2 P_{\text {in }}$ [30], therefore the proposed device acts as an all-optical amplitude jitter magnifier at this power. It is also noteworthy that for an input working power of $3.2 \mathrm{~W}$, the system exhibits the optical functionality of a bit-error indicating scheme $[31,32]$. 

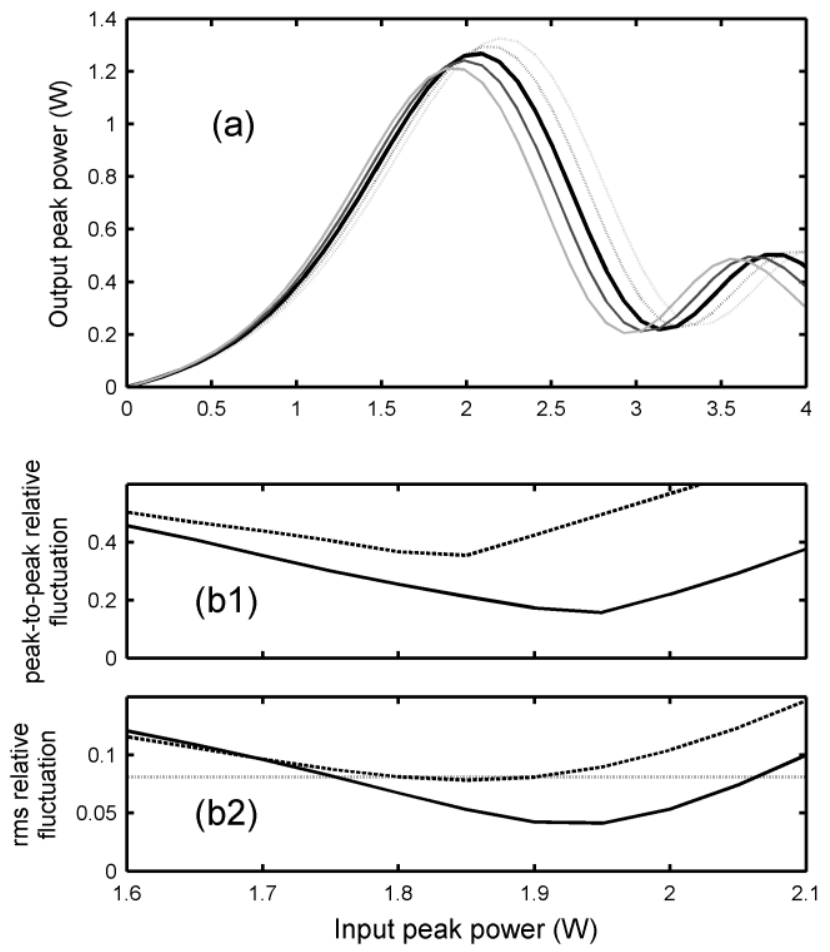

Figure 4: (a) TF of the device for initial pulses without pulse width variation (black) and with pulse width relative standard deviations of $-1,-2,+1$ and $+2 \%$ (grey). (b) Peak-to-peak and rms variations of the output pulse peak power versus the input power for an initial amplitude jitter with $8 \%$ relative standard deviation. The results obtained in the absence of initial ASE noise (solid) are compared with those in the presence of noise (17-dB OSNR; dotted).

In order to assess the regeneration capability of the device, we tested its operation with a RZ amplitude-shift keying pulse train modeled by a pseudorandom binary sequence of length 4096 bits. We illustrate the results with a pulse train with a repetition rate set to $500 \mathrm{MHz}$, whilst the pulse width was kept constant to $2 \mathrm{ps}$. We confirmed similar trends to the ones described hereinafter are observed for pulse repetition rates up to $10 \mathrm{GHz}$, which prevent the occurrence of pulse-to-pulse interactions during nonlinear propagation in the fibre [33, 34]. Firstly, we considered the effect of amplitude fluctuations in the one-bit pulses alone. The signal was impaired by an rms amplitude jitter of $8 \%$ of ideal power and a finite extinction ratio (defined here as the ratio of the mean peak power of the one-bit pulses to the peak power of the zero-bit pulses). In Fig. 4(b), we show the fluctuations in output peak power of the marks as functions of the input power (solid line). As expected, optimum operation of the device is obtained for an input power close to the location of the maximum of the TF curve $(1.95 \mathrm{~W})$. At this working power, the rms variation of the output power is reduced to half of the input value. In Fig. 5(a), 
we compare the eye diagram of the signal at the output of the regenerator with the eye pattern of the input signal after linear filtering by the OBPF. The performance improvements achieved by the nonlinear stage of spectral compression are readily apparent: reduced amplitude jitter in the one-bit pulses and background level in the zero-bit slots, and a nearly fourfold increase in the one-bit pulse level reflecting in an improved extinction ratio. The statistical distribution of the peak power of the one-bit pulses (Fig. 4(b)) highlights the reshaping of the amplitude fluctuations accomplished by the spectral compression stage: at the regenerator output the distribution has no longer a Gaussian shape and is highly skewed to the left. We note that in the case of probability density functions of essentially non-Gaussian shapes, the conventional (Gaussian-based) Q-factor of the received signal [35] can only be used as a measure of the signal quality, whereas predictions of the bit-error rate based on this method can yield incorrect results.
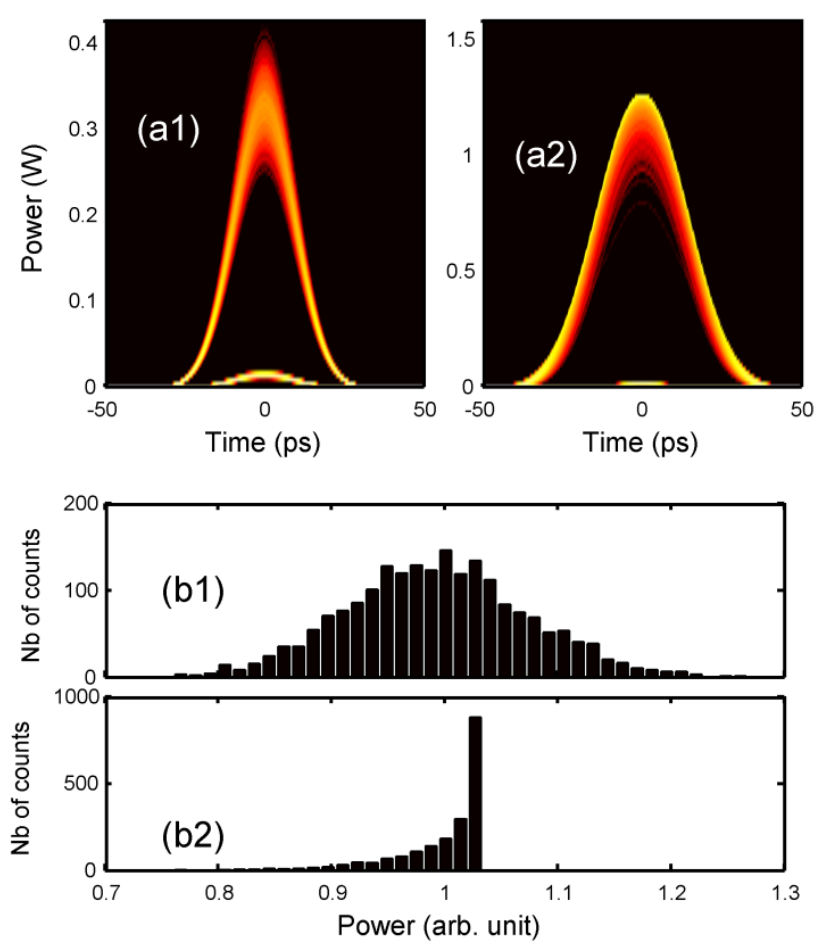

Figure 5: (a) Eye diagrams of the input 4096 pseudorandom binary pulse train filtered by a 20-GHz OBPF (subplot 1) and the pulse train at the regenerator output (subplot 2). (b) Distributions of the one-bit pulse level at the input (subplot 1) and output (subplot 2) of the regenerator. An rms amplitude jitter of $8 \%$ of ideal power is superimposed on the initial pulse train. 

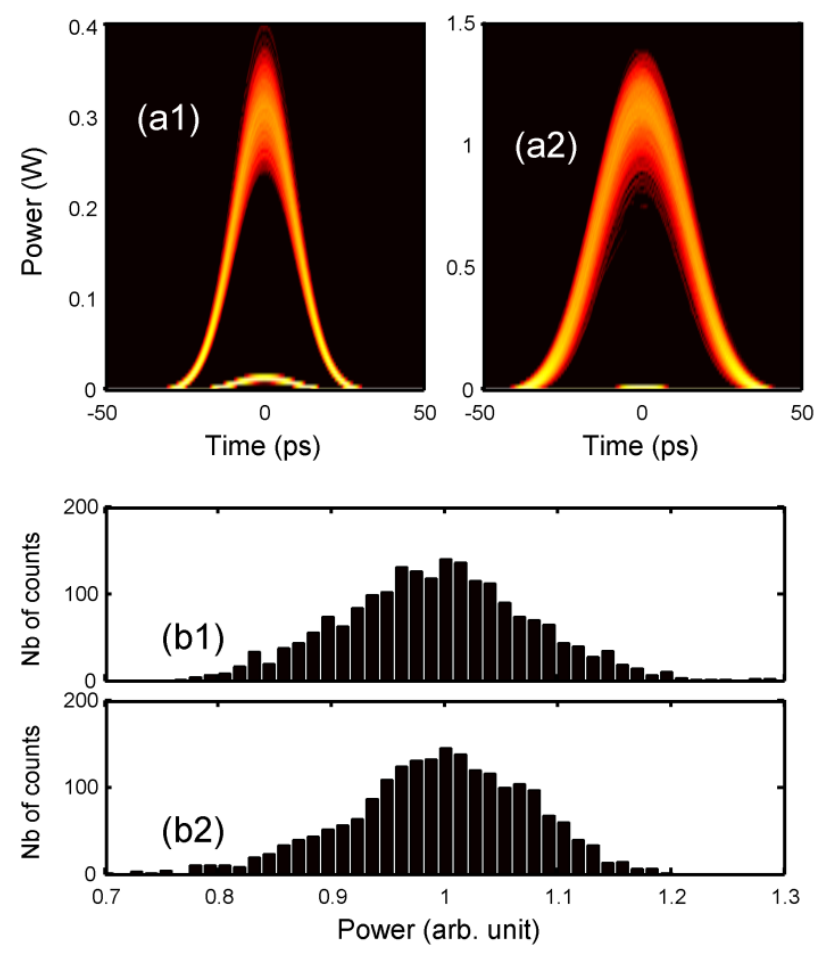

Figure 6: (a) Eye diagrams of the input 4096 pseudorandom binary pulse train filtered by a 20-GHz OBPF (subplot 1) and the pulse train at the regenerator output (subplot 2). (b) Distributions of the on-bit pulse level at the input (subplot 1) and output (subplot 2) of the regenerator. The initial pulse train is impaired by an rms amplitude jitter of $8 \%$ of ideal power and an OSNR of $17 \mathrm{~dB}$.

Next, we evaluated the combined effects of amplitude fluctuations and OSNR degradation of the initial pulse train. Figures 4(b1)-(b2) and 6 summarize the results obtained for an initial OSNR value of $17 \mathrm{~dB}$. In this case, the optimum working input power is slightly changed and is now close to $1.85 \mathrm{~W}$. Remarkably, the efficiency of the amplitude fluctuation mitigation has lessened so that even at the point of optimum operation, the output amplitude jitter is similar to the input one. The eye diagrams and statistical distributions in Fig. 6 confirm the impact of OSNR on the regeneration process. Even though improvement of the extinction ratio is still observed, the statistical distribution of the one-bit level at the regenerator output maintains a Gaussian shape but is narrower than the input distribution. The improvement in signal quality is therefore marginal. We attribute this reduction of the device's performance to the influence of the timing jitter incurred by the input pulses because of the added ASE noise. Indeed, as shown in Fig. 4(a) and already demonstrated in the Mamyshev's regenerator configuration [34], 
contrary to other nonlinear regenerators such as nonlinear optical loop mirrors, whose TFs only depend on the instantaneous pulse power [36], a variation in the input pulse duration affects the TF of our device by shifting the optimum operational power, thus dramatically impacting its beneficial effect.

\section{Conclusion}

We have studied the stability of the process of spectral compression arising from nonlinear pulse propagation in an optical fibre against amplitude fluctuations and a degraded OSNR of the seed pulses. We have shown that the nonlinear process in the regime of normal dispersion is rather stable against these pulse degradation factors, even though some portions of the compressed spectrum may be affected by an amplification of fluctuations. Combining spectral compression with an OBPF centered at the same frequency as the signal, the scheme enables partial suppression of the amplitude noise in an intensity-modulated signal. However, in the presence of a jitter of the temporal duration of the pulses, the performance of the device deteriorates.

A natural extension of this work would include the use of parabolic pre-shaping of the pulses [7, 11] or of an additional phase modulation [17] possibly restraining detrimental unstable oscillations of the spectrum, to improve the proposed nonlinear processing scheme. The benefits

of the spectral compression occurring upon pulse propagation in a dispersive-nonlinear regime $[15,16]$ in terms of enhanced opening of a signal eye pattern could also be explored. Using time interleaving, the scheme should be suitable for multi-wavelength operation $[37,38]$. It can also potentially be compatible with a simultaneous processing of the state of polarization of a signal [39]. We also believe that our results, obtained in the context of fibre optics, can be readily extended to highly nonlinear waveguides $[8,40]$ to achieve on-the-chip functions. Moreover, we expect that the present study can support a better understanding of the stability of multistage fibre amplifier architectures as well as their practical tolerance to pulse degradation factors [13, 41] as well as provide a deeper insight into the in-cavity dynamics of mode-locked fibre lasers $[14,42]$. 


\section{Acknowledgements}

We acknowledge financial support by the Conseil Regional de Bourgogne (Pari Photcom) and the Agence Nationale de la Recherche (Labex ACTION program ANR-11-LABX-01-01).

\section{References}

[1] R.H. Stolen, C. Lin, Self-phase modulation in silica optical fibers, Phys. Rev. A, 17 (1978) 1448-1453.

[2] A.V. Zohrabian, L.K. Mouradian, Compression of the spectrum of picosecond ultrashort pulses, Quantum Electronics, 25 (1995) 1076.

[3] M. Oberthaler, R.A. Höpfel, Spectral narrowing of ultrashort laser pulses by self-phase modulation in optical fibers, Appl. Phys. Lett., 63 (1993) 1017-1019.

[4] S.A. Planas, N.L. Pires Mansur, C.H. Brito Cruz, H.L. Fragnito, Spectral narrowing in the propagation of chirped pulses in single-mode fibers, Opt. Lett., 18 (1993) 699-701.

[5] B.R. Washburn, J.A. Buck, S.E. Ralph, Transform-limited spectral compression due to selfphase modulation in fibers, Opt. Lett., 25 (2000) 445-447.

[6] D.A. Sidorov-Biryukov, A. Fernandez, L. Zhu, A. Pugzlys, E.E. Serebryannikov, A. Baltuska, A.M. Zheltikov, Spectral narrowing of chirp-free light pulses in anomalously dispersive, highly nonlinear photonic-crystal fibers, Opt. Express, 16 (2008) 2502-2507.

[7] E.R. Andresen, C. Finot, D. Oron, H. Rigneault, Spectral Analog of the Gouy Phase Shift, Phys. Rev. Lett., 110 (2013) 143902.

[8] C. Mei, J. Yuan, K. Wang, X. Sang, C. Yu, Chirp-free Spectral Compression of Parabolic Pulses in Silicon nitride Channel Waveguides, 21st Optoelectronics and Communications Conference / International Conference on Photonics in Switching, Japan, 2016, pp. WE1-5.

[9] M. Rusu, O.G. Okhotnikov, All-fiber picosecond laser source based on nonlinear spectral compression, Appl. Phys. Lett., 89 (2006) 091118.

[10] J.P. Limpert, T. Gabler, A. Liem, H. Zellmer, A. Tünnermann, SPM-induced spectral compression of picosecond pulses in a single-mode Yb-doped fiber amplifier, Appl. Phys. B, 74 (2002) 191-195.

[11] J. Fatome, B. Kibler, E.R. Andresen, H. Rigneault, C. Finot, All-fiber spectral compression of picosecond pulses at telecommunication wavelength enhanced by amplitude shaping, Appl. Opt., 51 (2012) 4547-4553.

[12] C. Bao, X. Xiao, C. Yang, Spectral compression of a dispersion-managed mode-locked Tm:fiber laser at 1.9 um, IEEE Photon. Technol. Lett., 28 (2015) 497-500.

[13] D.A. Korobko, O.G. Okhotnikov, I.O. Zolotovskii, Multistage fiber preamplifier comprising spectral compression for generation of high-energy laser pulses J. Opt. Soc. Am. B, 33 (2016) 239-245.

[14] S. Boscolo, S.K. Turitsyn, C. Finot, Amplifier similariton fiber laser with nonlinear spectral compression, Opt. Lett., 37 (2012) 4531-4533. 
[15] A.A. Kutuzyan, T.G. Mansuryan, G.L. Esayan, R.S. Akobyan, L.K. Mouradian, Dispersive regime of spectral compression, Quantum Electronics, 38 (2008) 383-387.

[16] C. Finot, S. Boscolo, Design rules for nonlinear spectral compression in optical fibers, J. Opt. Soc. Am. B, 33 (2016) 760-767.

[17] S. Boscolo, L.K. Mouradian, C. Finot, Enhanced nonlinear spectral compression in fibre by external sinusoidal phase modulation, J. Opt, 18 (2016) 105504.

[18] G.P. Agrawal, Nonlinear Fiber Optics, Fourth Edition, Academic Press, San Francisco, CA, 2006.

[19] A.P. Grigoryan, Impact of coherency on the process of spectral compression of randomly modulated pulses, Proceedings of the YSU, Physics \& Mathematics, 2 (2015) 53-56

[20] S.K. Turitsyn, A.E. Bednyakova, M.P. Fedoruk, S.B. Papernyi, W.R.L. Clements, Inverse four-wave mixing and self-parametric amplification in optical fibre, Nat Photon, 9 (2015) 608614.

[21] E.R. Andresen, J. Thogersen, S.R. Keiding, Spectral compression of femtosecond pulses in photonic crystal fibers, Opt. Lett., 30 (2005) 2025-2027.

[22] J.P. Limpert, A. Liem, T. Gabler, H. Zellmer, A. Tünnermann, S. Unger, S. Jetschke, H.-R. Müller, High-average-power picosecond Yb-doped fiber amplifier, Opt. Lett., 16 (2001) 18491851.

[23] S. Taccheo, K. Ennser, Investigation of amplitude noise and timing jitter of supercontinuum spectrum-sliced pulses, IEEE Photon. Technol. Lett., 14 (2002) 1100-1102.

[24] P.V. Mamyshev, All-optical data regeneration based on self-phase modulation effect, European Conference on Optical Communication, ECOC'98, Institute of Electrical and Electronics Engineering, Madrid, Spain, 1998, pp. 475-476.

[25] H. Murai, Y. Kanda, M. Kagawa, S. Arahira, Regenerative SPM-based wavelength conversion and field demonstration of 160-Gb/s all-optical 3R operation, J. Lightw. Technol., 28 (2009) 910-921.

[26] M. Asobe, A. Hirano, Y. Miyamoto, K. Sato, K. Hagimoto, Y. Yamabayashi, Noise reduction of $20 \mathrm{Gbit} / \mathrm{s}$ pulse train using spectrally filtered optical solitons, Electron. Lett., 34 (1998) 1135-1136.

[27] M. Gay, M. Costa e Silva, T.N. Nguyen, L. Bramerie, T. Chartier, M. Joindot, J.C. Simon, J. Fatome, C. Finot, J.L. Oudar, $170 \mathrm{Gbit} / \mathrm{s}$ bit error rate assessment of regeneration using a saturable absorber and a nonlinear fiber based power limiter, IEEE Photon. Technol. Lett., 22 (2010) 158-160.

[28] L. Provost, C. Finot, K. Mukasa, P. Petropoulos, D.J. Richardson, Design scaling rules for 2R-Optical Self-Phase Modulation-based regenerators 2R regeneration, Opt. Express, 15 (2007) 5100-5113.

[29] J. Fatome, C. Finot, Scaling guidelines of a soliton-based power limiter for 2R-optical regeneration applications, J. Lightw. Technol., 28 (2010) 2552-2556.

[30] C. Finot, J. Fatome, All-optical fiber-based ultrafast amplitude jitter magnifier, Opt. Express, 18 (2010) 18697-18702.

[31] Z. Zhang, X. Zhou, R. Liang, Z. Qin, Y. Liu, An all-optical bit-error indicating scheme based on self-phase modulation and filtering, Opt. Commun., 282 (2009) 3163-3167.

[32] C. Finot, J. Fatome, Experimental demonstration of an ultrafast all-optical bit-error indicating scheme, Microw. Opt. Technol. Lett., 53 (2010) 392-395.

[33] T.N. Nguyen, T. Chartier, L. Bramerie, M. Gay, Q.T. Le, S. Lobo, M. Joindot, J.C. Simon, J. Fatome, C. Finot, Self-phase-modulation-based 2R regenerator including pulse compression 
and offset filtering for 42.6 Gbit/s RZ-33\% transmission systems, Opt. Express, 17 (2009) 17747-17757.

[34] C. Finot, T.N. Nguyen, J. Fatome, T. Chartier, L. Bramerie, M. Gay, S. Pitois, J.C. Simon, Numerical study of an optical regenerator exploiting self-phase modulation and spectral offset filtering at $40 \mathrm{Gbit} / \mathrm{s}$, Opt. Commun., 281 (2008) 2252-2264.

[35] G.P. Agrawal, Fiber-Optic Communication Systems, Wiley-Interscience2002.

[36] S. Boscolo, S.K. Turitsyn, K.J. Blow, Nonlinear loop mirror-based all-optical signal processing in fiber-optic communications, Opt. Fiber. Technol., 14 (2008) 299-316.

[37] T. Ohara, H. Takara, A. Hirano, K. Mori, S. Kawanishi, 40 Gb/s x 4 Channel all-optical multichannel limiter utilizing spectrally filtered optical solitons, IEEE Photon. Technol. Lett., 15 (2003) 763-765.

[38] L. Provost, F. Parmigiani, C. Finot, K. Mukasa, P. Petropoulos, D.J. Richardson, Analysis of a two-channel 2R all-optical regenerator based on a counter-propagating configuration, Opt. Express, 16 (2008) 2264-2275.

[39] P. Morin, J. Fatome, C. Finot, S. Pitois, R. Claveau, G. Millot, All-optical nonlinear processing of both polarization state and intensity profile for $40 \mathrm{Gbit} / \mathrm{s}$ regeneration applications, Opt. Express, 19 (2011) 17158-17166.

[40] V.G. Ta'eed, M. Shokooh-Saremi, L. Fu, D.J. Moss, M. Rochette, I.C.M. Littler, B.J. Eggleton, Y. Ruan, B. Lutther-Davies, Integrated all-optical pulse regenerator in chalcogenide waveguides, Opt. Lett., 30 (2005) 2900-2902.

[41] S. Wang, W. Chen, P. Qin, Y. Song, M. Hu, B. Liu, Spectral and temporal breathing selfsimilar evolution in a fiber amplifier for low-noise transform-limited pulse generation, Opt. Lett, 41 (2016) 5286-5289.

[42] Y. Lan, Y. Song, M. Hu, B. Liu, L. Chai, C. Wang, Enhanced spectral breathing for sub-25 fs pulse generation in a Yb-fiber laser, Opt. Lett., 38 (2013) 1292-1294. 